\title{
Erratum
}

\section{Isolation of chalcone synthase and chalcone isomerase cDNAs from alfalfa (Medicago sativa L.): highest transcript levels occur in young roots and root tips}

\author{
Heather I. McKhann ${ }^{1}$ and Ann M. Hirsch* \\ Department of Biology, 405 Hilgard Ave., University of California, Los Angeles, Los Angeles, CA 90024, \\ USA (* author for correspondence); ${ }^{1}$ Present address: Institut des Sciences Végétales, Avenue de la Terrasse, \\ 91198 Gif-sur-Yvette, France
}

Plant Molecular Biology 24: 767-777. 1994.

These was an error in the above mentioned paper. In Fig. 1, part B, the legends for Medicago sativa and Medicago truncatula were reversed. The correct version is shown below:

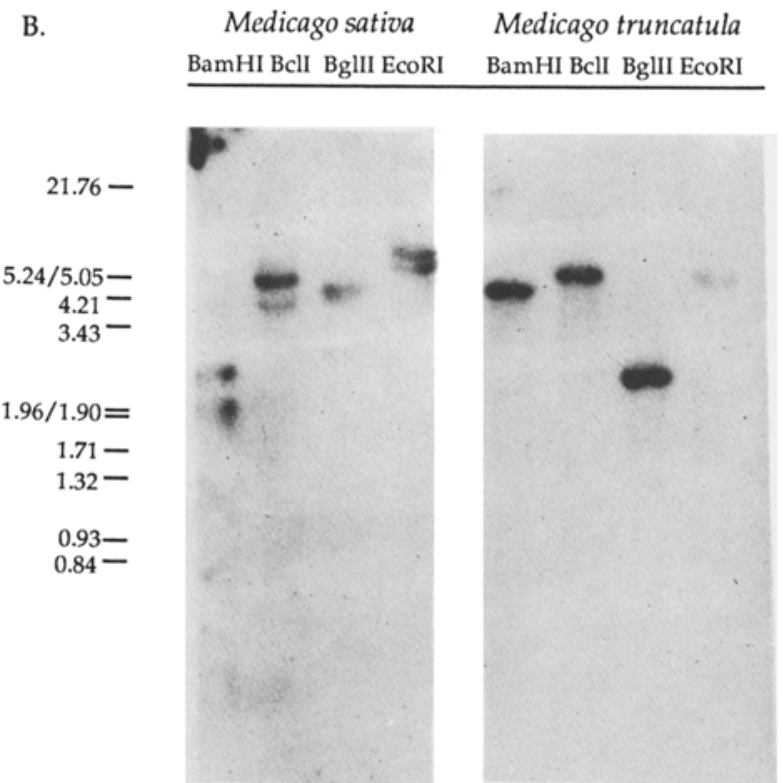

Fig. 1. Southern analysis of chalcone synthase and chalcone isomerase in alfalfa (Medicago sativa cv. Iroquois) and Medicago truncatula L. cv. Jemalong. Duplicate genomic Southern blots probed with full-length CHS4-1 probe (A) and CHI-1 probe (B). Restriction enzymes used are as indicated. Ca. $2 \mu \mathrm{g}$ DNA were loaded in each lane. 